\title{
Multimodal Stratified Approach for Prevention of Thromboembolism in Patients Undergoing Total Hip Arthroplasty
}

\author{
Elmano de Araújo Loures ${ }^{*}$, Glauco Mendonça Rocha², Daniel Naya Loures ${ }^{2}$, \\ Isabel Cristina Gonçalves Leite ${ }^{3}$, Clarice Naya Loures ${ }^{4}$
}

\author{
${ }^{1}$ University Hospital of the Federal University of Juiz de Fora, Juiz de Fora MG, Brasil \\ ${ }^{2}$ Rio Branco Medical Center, Juiz de Fora-MG, Brasil \\ ${ }^{3}$ School of Medicine of the Federal University of Juiz de Fora, Juiz de Fora-MG, Brasil \\ ${ }^{4}$ São Paulo Federal University, São Paulo-SP, Brasil \\ Email: *loures.elmano@gmail.com
}

How to cite this paper: Author 1, Author 2 and Author 3 (2018) Paper Title. Open Journal of Orthopedics, 8, 429-442. https://doi.org/10.4236/ojo.2018.812045

Received: July 23, 2018

Accepted: November 30, 2018

Published: December 3, 2018

Copyright (c) 2018 by authors and Scientific Research Publishing Inc. This work is licensed under the Creative Commons Attribution International License (CC BY 4.0).

http://creativecommons.org/licenses/by/4.0/

\begin{abstract}
Objective: To demonstrate the efficacy and safety of a multimodal stratified approach for prevention of thromboembolism in patients undergoing primary total hip arthroplasty. Method: A longitudinal retrospective clinical study of a cohort of 341 individuals undergoing total hip arthroplasty between March 2008 and July 2016. Of these, 242 patients met the criteria for inclusion in the study. Individuals with a history of deep vein thrombosis or pulmonary thromboembolism, thrombophilia, hypercoagulation conditions, and chronic users of anticoagulants before surgery received enoxaparin at a dose of $40 \mathrm{mg} /$ day for 21 days. For the other group, acetylsalicylic acid was prescribed at a dose of $200 \mathrm{mg} /$ day for 30 days. All patients had surgical risk assessed by the American Anesthesiology Association classified as I or II, and underwent regional anesthesia, effective hydration, the same protocol of early postoperative rehabilitation, and the use of compression stockings. Drug cost analysis was performed based on the Brasíndice. To date, there are no publications in PubMed and Scielo on this subject regarding the Brazilian population. Results: The need for blood transfusion in the enoxaparin group was higher and the overall complication and hemorrhagic rates were also significantly higher $(p<0.010)$ in this group. Aspirin prescription costs 39 times less than enoxaparin. Conclusion: In patients without predisposing factors to thromboembolism and undergoing primary total hip arthroplasty, the multimodal approach with acetylsalicylic acid seems to be effective, with low cost and a low rate of complications.
\end{abstract}

\section{Keywords}

Anticoagulation, Thromboembolism, Total Hip Arthroplasty 


\section{Introduction}

Total hip arthroplasty (THA) is one of the surgical procedures with a high success rate in orthopedic surgery, eliminating pain and restoring hip function in osteoarthrosis cases [1]. It is a surgical procedure with a major impact on the natural history of degenerative hip arthritis, providing the patient with a marked and rapid improvement in function and quality of life, assessed by specific scores such as the Harris Hip Score and the SF-36 [2] [3].

However, a number of local and systemic complications may occur. Thromboembolic phenomena are one of the main systemic complications. The incidence of deep vein thrombosis (DVT) may reach $15 \%$ in these patients, even with preventive measures and pulmonary thromboembolism (PE) occurs in $1 \%$ to $3 \%$ of cases, with up to $50 \%$ mortality [4]. Various alternatives have been proposed in search of the best method capable of reducing the incidence of DVT/PE, recognized as preventable events. The use of several anticoagulant agents such as warfarin, heparin and enoxaparin and an aggressive chemical prophylaxis were the standard of care for all patients during two decades associated to the use of elastic compression stockings and early mobilization. More recently, oral drugs dabigatran and rivaroxaban were also introduced with the same purposes. Preventing symptomatic and/or fatal pulmonary thromboembolism is the main objective of prophylaxis [5].

The guidelines of the American College of Chest Physicians (ACCP) which emphasized prophylaxis with potent anticoagulant agents, did not modify the rate of symptomatic or fatal pulmonary thromboembolism over more than a decade. In addition, they increased morbidity in the major orthopedic surgery setting, mainly related to hemorrhagic complications, thus motivating the search and the proposal for alternative protocols [6].

Several authors relate the use of anticoagulants to a higher rate of complications from excessive bleeding and infections, as well as the need for blood transfusion [7] [8]. The constant search for the best method and/or most effective drug with less undesirable effects is the subject of intense debate over new alternatives which have been changing the traditionally accepted guidelines [9]. Prophylaxis based on a set of mechanical measures and on the use of aspirin in patients who do not present risk factors for developing DVT and PE, has been gaining increasing credibility, anchored in large studies with high scientific impact, although there is still no consensus about the best chemical agent for thromboprophylaxis after THA [10].

In 2006, the American Academy of Orthopaedic Surgeons (AAOS) created a task force to define strategies for the prevention of thromboembolic phenomena in patients undergoing major orthopedic surgery. Since then, the understanding on the subject has been changing substantially [11].

Salvati et al. [12] emphasized the efficacy and safety of a multimodal approach using acetylsalicylic acid in patients with no predisposing factors to DVT/PE and who can be mobilized early. Intraoperative tactics are also recommended for the 
reduction of femoral instrumentation time, combined with continuous irrigation and suction [13].

Johanson et al. [14] corroborate this paradigm shift as it provides the orthopedic surgeon with a wider range of options and allows for the selection of less aggressive protocols. Thus, an equivalent level of efficacy and safety in the prevention of thromboembolic events, combined with the reduction of the undesirable effects of intensive anticoagulation is maintained [12].

The objective of the present study was to evaluate the efficacy and safety of the stratified multimodal approach using acetylsalicylic acid as chemoprophylaxis for DVT and PE in patients submitted to primary THA and to measure the incidence of thromboembolism and complications in the sample studied.

\section{Material and Methods}

The study was submitted to the institution's Research Ethics Committee under CAAE No. 13710914.6.0000.5133/opinion 779.089 and all the individuals selected explicitly agreed to participate by means of a free and informed consent form.

In a longitudinal retrospective clinical study, a cohort of 341 patients was analyzed. They had undergone primary or revision THA, for any diagnosis, between March 2008 and July 2016, were operated on by the same senior surgeon, using the lateral approach [15], in 3 general hospitals, one a teaching hospital. The mean hospital stay after surgery was 72 hours. Of these, 242 patients fulfilled the criteria for inclusion in the study, that is, patients who had undergone total primary hip arthroplasty, with an ASA (American Society of Anesthesiologists) I or II surgical risk [16] and monitored for a period of 6 months.

Among those excluded from the analysis were 48 patients who had undergone revision THA surgery, 17 patients with incomplete documentation, 25 individuals for whom a postoperative suction drain was used, and 9 patients with a body mass index (BMI) of 30 or higher.

Blood transfusion of two units of packed red blood cells was indicated in cases with a hemoglobin level below $9 \mathrm{~g} / \mathrm{dl}$, collected 24 hours after the procedure.

Individuals were divided into two groups: one group composed of patients with a personal history of DVT or PE, thrombophilia, hypercoagulability, and chronic anticoagulant users prior to surgery, who received enoxaparin at a dose of $40 \mathrm{mg}$ day for 21 days, commencing 12 hours after the surgical procedure (Group I-56 individuals). For the rest, acetylsalicylic acid was administered at a dose of $200 \mathrm{mg} /$ day for a period of 30 days, commencing 12 hours after the surgical procedure (Group II-186 individuals). All were submitted to regional anesthesia, to effective hydration, to the same early postoperative rehabilitation protocol with mobilization in bed beginning in the sixth postoperative hour, to the use of elastic compression stockings, and intraoperative precautions to reduce the time of femoral instrumentation and extreme limb positions.

The cost study of the drugs used, acetylsalicylic acid $200 \mathrm{mg}$ for 30 days and 
enoxaparin $40 \mathrm{mg}$ for 21 days, was performed based on the mean consumer price published in the Brasíndice, December 2016 edition [17].

The data analysis was developed using the Statistical Package for the Social Sciences (SPSS), version 15.0 (Chicago, IL, USA). Incidence measurements were obtained for events after 180 days of follow-up. The quantitative variables were described in terms of means and standard deviations.

In the bivariate analysis, the chi-square test was used for nominal variables, with Fischer's correction when necessary, and crude odds ratios were obtained. Quantitative variables were analyzed via $t$-test for meeting the normal data distribution criterion.

The logistic regression analysis sought to control the effect of confounding variables and estimated the adjusted odds ratios, with model goodness-of-fit assessed by the Hosmer and Lemeshow method. The significance level was $5 \%$.

The term "hemorrhagic complication" was used, encompassing hematomas and/or serohematic drainage from the wound for more than 5 days.

\section{Results}

Fifty-six individuals (23.10\%) comprised group I with administration of enoxaparin.

One hundred and eighty-six individuals (76.09\%) were included in group II and used acetylsalicylic acid (Table 1).

Table 1. Demographic, diagnosis, and intervention variables, by drug used.

\begin{tabular}{|c|c|c|c|c|c|}
\hline \multirow[b]{2}{*}{ Variables } & \multicolumn{2}{|c|}{ Enoxaparin } & \multicolumn{2}{|c|}{ Aspirin } & \multirow[b]{2}{*}{$p$-value } \\
\hline & $\begin{array}{c}\text { Mean inc. } \\
(\%)\end{array}$ & SD & $\begin{array}{c}\text { Mean inc. } \\
(\%)\end{array}$ & $\mathrm{SD}$ & \\
\hline No. of patients & 23.10 & & 76.09 & & \\
\hline Mean age & 68.79 & 10.12 & 57.87 & 11.94 & 0.110 \\
\hline Male gender & 35.70 & & 57.50 & & 0.030 \\
\hline Type of prosthesis & & & & & 0.714 \\
\hline Hybrid & 62.50 & & 61.80 & & \\
\hline Non-cemented & 32.10 & & 29.60 & & \\
\hline Cemented & 5.40 & & 8.60 & & \\
\hline Re-intervention & 8.90 & & 1.60 & & 0.007 \\
\hline Transfusion & 25.00 & & 5.40 & & $<0.010$ \\
\hline Diagnosis & & & & & 0.310 \\
\hline Osteoarthrosis & 66.10 & & 58.60 & & \\
\hline Osteonecrosis & 7.10 & & 18.30 & & \\
\hline Dysplasia & 7.10 & & 8.10 & & \\
\hline Metabolic disorders & 7.10 & & 3.80 & & \\
\hline Legg-Perthes/epiphysolysis & 0.00 & & 1.60 & & \\
\hline Ottopelvis & 0.00 & & 1.10 & & \\
\hline Fractures & 12.50 & & 8.60 & & \\
\hline
\end{tabular}

Legend: Mean inc.-Meanincidence; SD—Standard Deviation. 
Of the patients in group I, $35.70 \%$ were male and $64.30 \%$ female. In group II, $57.50 \%$ were male and $42.50 \%$ were female, with a $p$-value $=0.030$ (Figure 1 ). The description of the sample according to demographic, diagnosis, and intervention characteristics is shown in Table 1.

The mean age of patients in group I was 68.79 years (SD 10.12) and in group II was 57.87 years (SD 11.94), $p$-value $=0.110$. The distribution is depicted in Figure 2.

The mean age of the group that received injectable anticoagulant was higher than the group that was given acetylsalicylic acid (68.79 years and 57.87 years, respectively), although not statistically significant ( $p$-value of 0.110 ). The female gender predominated in the enoxaparin group ( $p$-value of 0.030 ) (Figure 2).

Figure 3 shows the etiological diagnosis of the sample studied, and the distribution by etiological diagnosis according to the drug used is shown in Table 1, with no significant difference between the groups.

Regarding the type of implant used, $62.5 \%$ of the cases that used enoxaparin and $61.80 \%$ of those who used acetylsalicylic acid received a hybrid implant (cemented femoral stem). The non-cemented THA was used in $32.10 \%$ of the cases in group I and $29.60 \%$ in group II. Cemented prostheses were used in $5.40 \%$ of the individuals in group I and $8.60 \%$ in group II (Table 1 ).

The incidence of complications was higher for the enoxaparin group, with statistical significance $(p$-value $<0.001)$. The variables analyzed and the most frequent types of complications are listed in Table 2, covering a period of 180 days of postoperative follow-up.

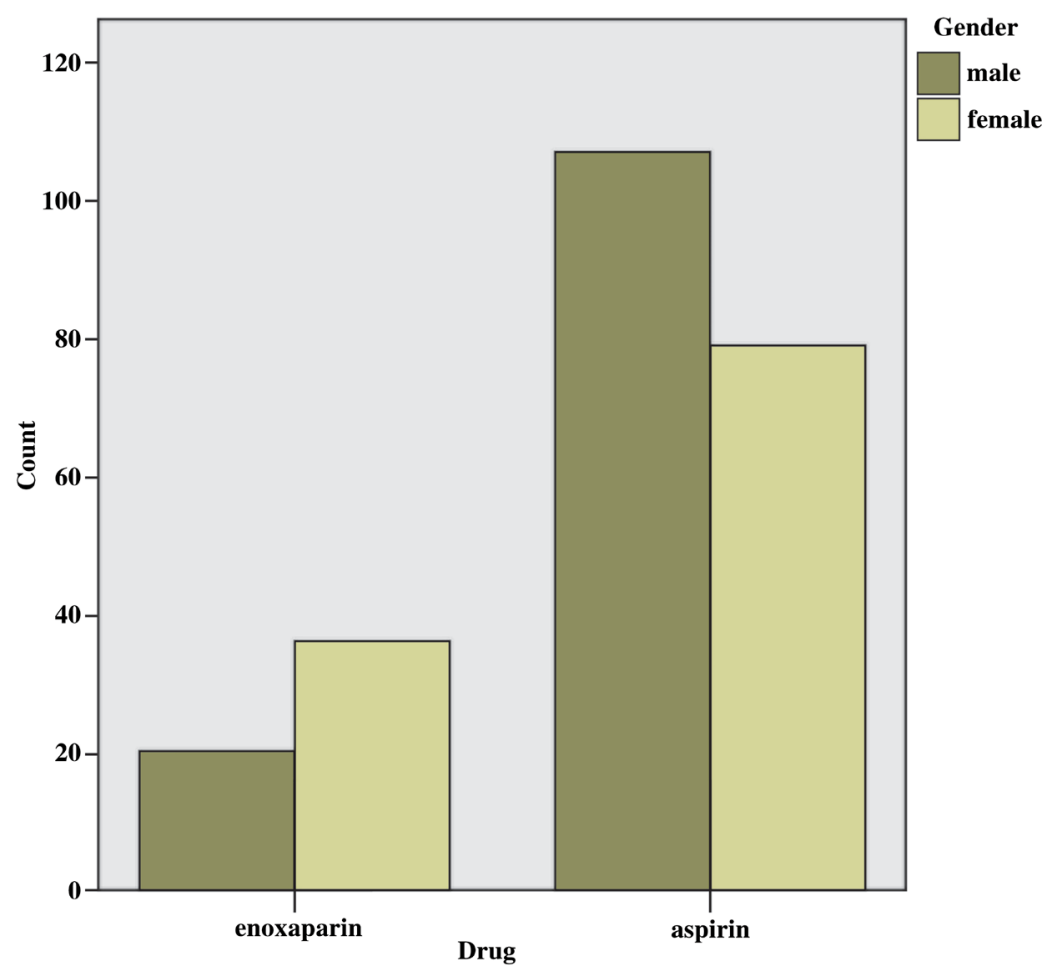

Figure 1. Distribution of the gender of the patients analyzed by type of drug used. 


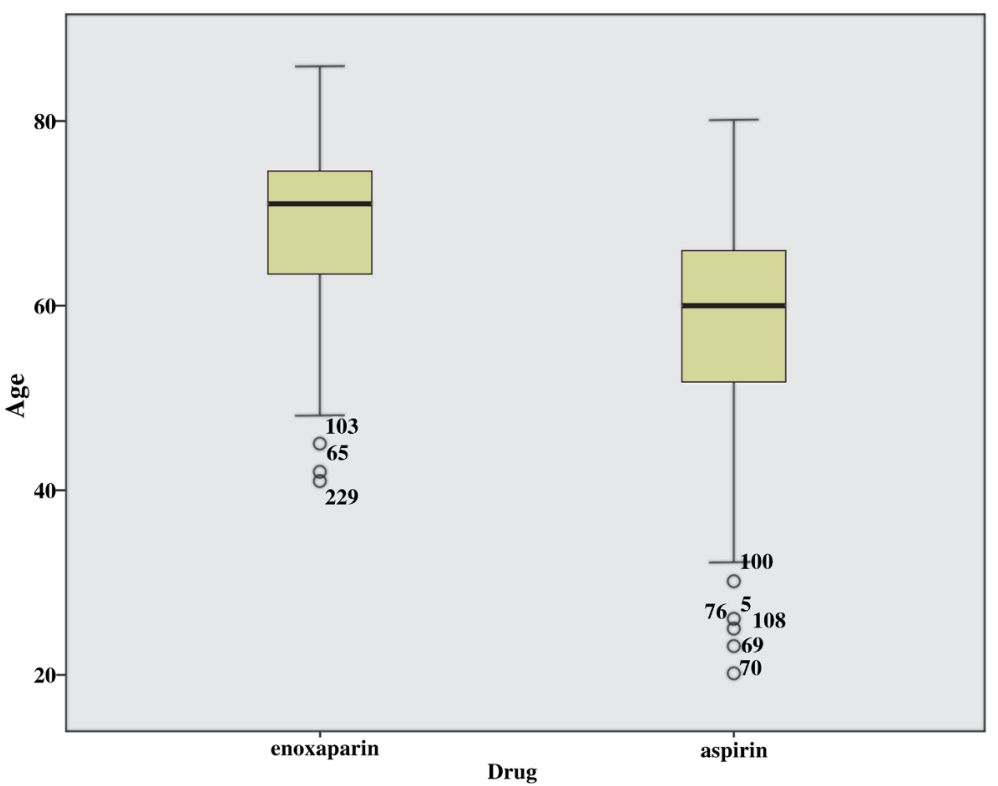

Figure 2. Mean age of the patients analyzed by type of drug used.

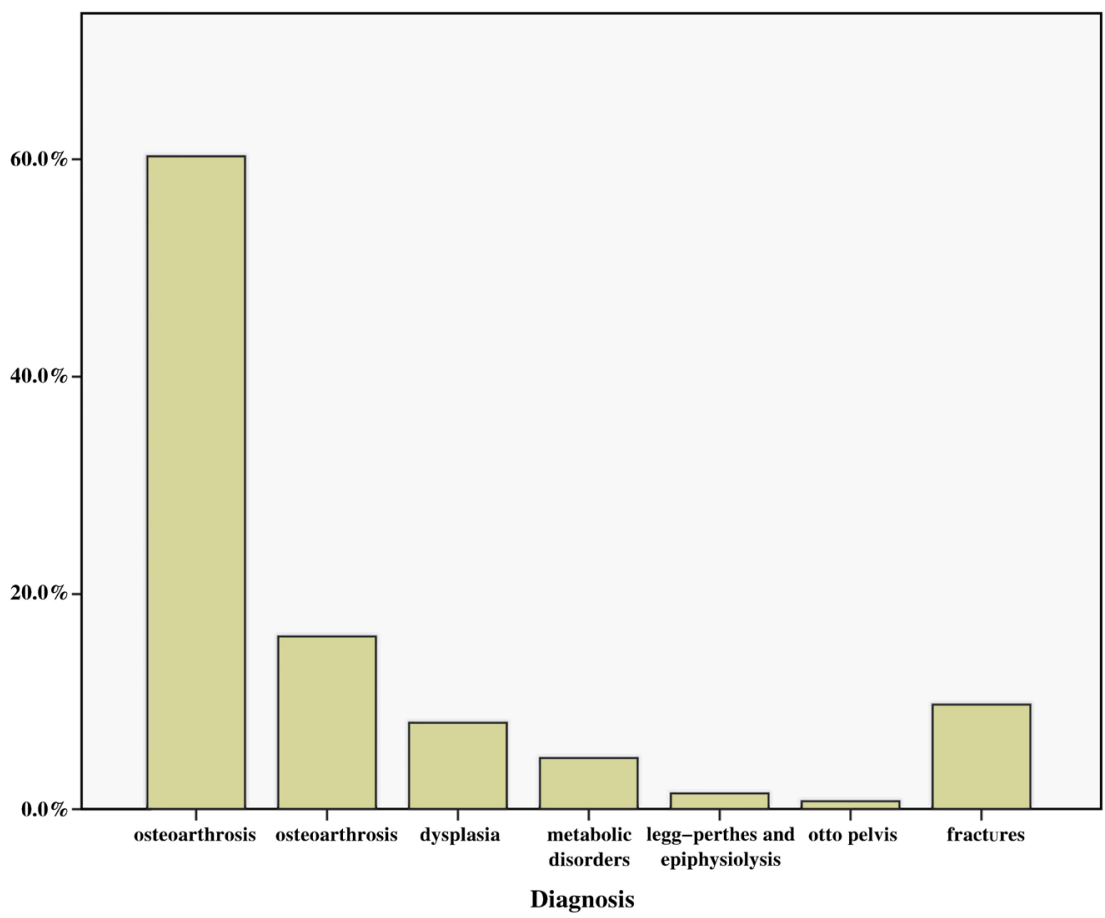

Figure 3. Distribution of the patients analyzed by etiological diagnosis.

The multivariate analysis, for the occurrence of complications with enoxaparin use, described in Table 3 , showed statistical significance $(p$-value $<0.010)$ for the variables "any complication" and "hemorrhagic complication".

Re-interventions for any reason were necessary in $8.90 \%$ of the cases in group I (enoxaparin) and $1.60 \%$ of the cases in group II (acetylsalicylic acid), with a $p$-value $=0.007$. 
Table 2. Complications according to the drug used (180 days).

\begin{tabular}{cccc}
\hline \multirow{2}{*}{ Variables } & \multicolumn{2}{c}{ Drug used } & \multirow{2}{*}{-value } \\
\cline { 2 - 3 } & Enoxaparin (\%) & Aspirin (\%) & \\
\hline Complication & 23.2 & 5.9 & $<0.001$ \\
Infection & 1.8 & 2.2 & \\
Neurapraxia & 0.0 & 0.5 & \\
Intra-operative fracture & 1.8 & 1.1 & \\
Post-operative drainage & 3.6 & 1.5 & \\
Hematoma & 7.1 & 0.0 & \\
Death & 1.8 & 0.0 & \\
Post-thrombotic syndrome & 0.0 & 0.5 & \\
Unconfirmed suspicion & 3.6 & 0.5 & \\
Deep vein thrombosis & 1.8 & 0.0 & \\
Pulmonary thromboembolism & 1.8 & 0.0 & \\
\hline
\end{tabular}

Table 3. Multivariate analysis* for the occurrence of selected complications and the use of enoxaparin.

\begin{tabular}{|c|c|c|c|}
\hline Variables & OR & CI $(95 \%)$ & $p$ value \\
\hline Any complication & 4.81 & $2.02-11.49$ & $<0.010$ \\
\hline Thrombotic complication $^{\dagger}$ & 4.44 & $3.51-5.69$ & 0.010 \\
\hline Hemorrhagic complication ${ }^{\ddagger}$ & 6.44 & $2.70-15.36$ & $<0.010$ \\
\hline Need for re-intervention & 5.98 & $1.38-25.90$ & 0.018 \\
\hline
\end{tabular}

Notes: ${ }^{*}$ Controlled for gender, mean age, and type of prosthesis; ${ }^{\dagger}$ Deep vein thrombosis and pulmonary thromboembolism; "Hematoma, serous drainage, and need for transfusion. Legend: OR-Odds Ratio; CI-Confidence Interval.

Blood transfusion was performed in $25.00 \%$ of the individuals in group I and $5.40 \%$ in group II, with a $p$-value $<0.010$ (Table 1 ). It was inferred that the use of enoxaparin represented an increased risk for blood transfusion and hemato$\mathrm{ma} /$ seroma formation and/or suspected infection.

The incidence of DVT was $1.8 \%$ and PE was $1.8 \%$ in group I. No cases were registered in group II (Table 2).

Suspected DVT not confirmed by venous Doppler was noted in $3.6 \%$ of the individuals in the enoxaparin group and $0.5 \%$ of the cases in the group that used acetylsalicylic acid. Post-thrombotic syndrome was identified in the 180-day follow-up in $0.5 \%$ of those who received acetylsalicylic acid.

The patients diagnosed with hip dysplasia had a higher need for blood transfusion (38\%) compared to the general rate $(p<0.0001)$. Such diagnosis therefore defined a possible risk factor for bleeding in the pre-operative period, a variable independent of the use of any of the drugs considered.

The mean cost of acetylsalicylic acid $200 \mathrm{mg}$, used for the 30-day period, was $\mathrm{R} \$ 26.04$ (26.04 Brazilian Reals) and of enoxaparin $40 \mathrm{mg}$, for the 21-day period, 
was R\$1073.05 (1073.05 Brazilian Reals).

\section{Discussion}

The use of potent anticoagulants in the pre and post-operative periods of orthopedic surgery has come under intense debate and is still a controversial issue. The increase in the risk of bleeding, blood transfusions, and clinical intercurrences are reported. On the other hand, the incidence of $\mathrm{PE}$ deaths has not changed over decades, even with the use of anticoagulants. The ACCP guidelines recommending potent anticoagulation tend to underestimate the risks of bleeding and its consequences and, until recently, contraindicated the use of acetylsalicylic acid as a single-agent drug in thromboprophylaxis [18] [19] [20] [21] [22].

The high incidence of complications in patients submitted to the ACCP protocol for the prevention of DVT/PE motivated the AAOS to propose new guidelines more appropriate to the orthopedic surgery scenario and its specifics, with the objective of preventing symptomatic PE and reducing the prevalence of DVT and, at the same time, minimizing the undesirable complications associated with more aggressive anticoagulation [12].

Burnett et al. [23], in a series of 290 prospectively evaluated patients and following the guidelines of the ACCP in the use of enoxaparin, found $9 \%$ of serious complications, DVT in $3.8 \%$, and nonfatal PE in $1.3 \%$, associated with a high rate of readmissions and interventions, prolonged drainage from the surgical wound, and 3.4\% of infections. They noted that a body mass index of 35 or higher is predictive of prolonged drainage from the wound, and that drainage of more than 7 days is predictive of readmission and reoperation.

Various publications in the last ten years advocate careful selection of patients and less aggressive conduct in the prevention of DVT/PE, with the use of simple protocols, with low cost and with lower risks, yet effective in preventing such events [24].

Ogonda et al. [25] analyzed 11,459 individuals submitted to hip and knee arthroplasties, for whom acetylsalicylic acid was the primary agent for thromboprophylaxis, and they support the individualized risk approach as part of a multimodal strategy. They concluded that acetylsalicylic acidis safe as a thromboprophylactic agent in arthroplasties and was not associated with an increased incidence of DVT, PE, or death. The main causes of death were ischemic heart disease and respiratory disorders, with PE accounting for only $18 \%$ of the deaths, whose incidence in 90 postoperative days was $0.39 \%$. The incidence of PE after THA was $0.6 \%$, and $0.3 \%$ for DVT in their study.

The use of the multimodal protocol for the prevention of DVT/PE with elastic compression stockings, early mobilization and ambulation, plus the administration of acetylsalicylic acid in the postoperative period, has proven to be effective and with a lower risk of bleeding, according to various authors [26]. To date there are no published studies on the topic, involving the Brazilian population, in the PubMed and Scielo databases. 
Acetylsalicylic acid would even have effects in reducing the risk of arterial complications and on heterotopic ossification, being easy to administer and well tolerated by most patients. Its use is beneficial in the three elements of Virchow's Triad (venous stasis, endothelial lesion, and hypercoagulation), with rates of $2.5 \%$ for DVT, $0.6 \%$ for non-fatal PE and an absence of major bleeding events, as reported by González Della Valle et al. [27].

Salvati et al. [12] justify the multimodal approach owing to its lower rates of mortality, non-fatal PE and bleeding, forming an effective strategy with fewer complications when using an acetylsalicylic acid dose of $650 \mathrm{mg} /$ day [18].

Other authors [28] recommend doses below $300 \mathrm{mg} /$ day and, therefore, there is still no consensus regarding the most appropriate dose of acetylsalicylic acid.

The choice of an acetylsalicylic acid dose of $200 \mathrm{mg} / \mathrm{day}$ in the present sample was due to the practicality of administration with the most commonly offered medication available in the Brazilian market, being defined by the average between the dosages proposed by different authors.

In the present analysis, a homogeneity was observed between the two groups in most of the variables considered, and there were no significant differences in the demographic variables, in the diagnosis, and in the type of implant used, except for the mean age of the acetylsalicylic acid group being lower than that of the group who received enoxaparin, and the predominance of the male gender in the acetylsalicylic acid group.

The protocol was applied with no distinctions for the public network and private clinic patients.

The present study's data analysis demonstrated a greater need for blood transfusion in patients undergoing anticoagulation with enoxaparin $(p<0.010)$ and a higher risk of bleeding associated with the presence of hematoma/seroma in the same group (OR 6.44; CI $2.70-15.36$; $p$-value $<0.010$ ), data in agreement with the literature researched.

Some authors report that the greater volume of drainage from the surgical wound in the postoperative period was related to an increased risk of infection of $42 \%$ in patients submitted to THA and $29 \%$ in patients submitted to total knee arthroplasty (TKA) [29] [30]. In this series, statistical significance was not attained in relation to the prevalence of infection. However, the group receiving enoxaparin had a higher rate of reintervention $(p$-value $=0.007)$ and postoperative drainage with OR 4.06 (0.56 - 29.41).

In a study with 473 low-risk patients for the development of DVT/PE who underwent total knee arthroplasty and were treated with a multimodal protocol and acetylsalicylic acid, Callaghan et al. reported the non-occurrence of death and a very low cost to carry out the prevention of thromboembolic phenomena [31].

The only recorded death in the sample under analysis was due to a coronary event in the first 12 hours postoperatively.

Adherence to treatment also seems to be associated with the cost of the drug. It is observed that this is one of the main reasons for not adhering to the pre- 
scribed medication use in the correct way and for the time required, especially among the most needy population attended by the Brazilian Unified Health System (SUS). The extremely high financial cost of enoxaparin (R $\$ 1073.05)$ compared to acetylsalicylic acid ( $\mathrm{R} \$ 26.04)$, for the period of 21 and 30 days respectively [17], clearly favors the option for using the latter [32].

Johanson et al. [14], in the guidelines recommended by the AAOS in 2009, affirm it is advisable to evaluate the risk not only of thromboembolic events, but also of postoperative bleeding, and that mechanical prophylactic measures with early mobilization are recommended to all individuals who undergo hip or knee arthroplasties, without distinctions. They also highlight the recommendations on drug selection for different groups of patients. For individuals with a normal risk for thromboembolism and/or a high risk for bleeding, the choice of aspirin is supported. For individuals with a high risk of thromboembolism and a normal level of risk for bleeding, low molecular weight heparin, or other more potent agents, also have an established role.

It was also observed in this series that patients with a diagnosis of hip dysplasia presented a higher risk of complications related to bleeding.

It should be noted that the use of aspirin does not require periodic control of the International Normalized Ratio (INR), further reducing the secondary additional costs and facilitating the follow-up of cases. Likewise, the increasing number of younger-age-group individuals favors the use of less aggressive protocols for anticoagulation, combined even with the fact that most thromboembolic events occur on the first three postoperative days [22].

Recent changes in the guidelines of both the AAOS and the ACCP converge in favor of aspirin use in the context of a multimodal approach in correctly selected patients. Bayley et al. [33], in a prospective study involving 7983 THAs, suggest aspirin as an appropriate thromboprophylactic agent as part of a multimodal regimen, noting that pulmonary embolism is rare in this context and that potent anticoagulants have a higher rate of complications.

While noting the limitations of the present study in relation to the sample size, the low incidence of complications combined with the low cost of the aspirin administered for most cases $(76.09 \%)$ may represent a relevant indication for the prophylaxis strategy of DVT/PE in the postoperative period of THA in the Brazilian population, especially in the low-income group attended by the Unified Health System (SUS).

\section{Conclusions}

Data analysis showed a higher overall rate of complications such as bleeding and hematoma/seroma formation, as well as in the indication of blood transfusion in patients submitted to prevention of thromboembolic events with enoxaparin compared to those who received aspirin, with statistical significance. The financial cost of the multimodal approach with aspirin proved exponentially lower than the use of enoxaparin. 
Until further studies in a larger population, it is advisable to keep using with caution enoxaparin or other potent anticoagulants in selected individuals prone to develop DVT/PE in the THA postoperative.

In patients without predisposing factors to thromboembolism and having undergone primary THA, the multimodal approach using acetylsalicylic acid was effective, low-cost, with a low complication rate and no increase in the incidence of DVT/PE.

\section{Author Contributions}

Elmano de Araújo Loures-Substantial contributions to conception and design, Acquisition of data, Analysis and interpretation of data, Drafting the article, Revising it critically for important intellectual content, Final approval of the version to be published.

Glauco Mendonça Rocha-Substantial contributions to conception and design, Acquisition of data, Analysis and interpretation of data, approval of the version to be published.

Daniel Naya Loures-Substantial contributions to conception and design, Analysis and interpretation of data, Revising it critically for important intellectual content, Final approval of the version to be published.

Clarice Naya Loures-Substancial contributions to conception and design, Analysis and interpretation of data, revising it critically for important intellectual content, Final approval of the version to be published.

Isabel Cristina Goncalves Leite-Substancial contributions to conception and design, Analysis and interpretation of data, Final approval of the version to be published.

\section{Conflicts of Interest}

The authors of this study declare there was no conflict of interest.

\section{References}

[1] Pearcy, M.J. (1988) A New Generation of Artificial Hip Joints. Engineering in Medicine, 17, 199-201. https://doi.org/10.1243/EMED_JOUR_1988_017_049_02

[2] Laupacis, A., Bourne, R., Rorabeck, C., Feeny, D., Wong, C., Tugwell, P., et al. (1993) The Effect of Elective Total Hip Replacement on Health-Related Quality of Life. The Journal of Bone and Joint Surgery. American Volume, 75, 1619-1626. https://doi.org/10.2106/00004623-199311000-00006

[3] Loures, E., de, A. and Leite, I.C.G. (2012) Análise da qualidade de vida de pacientes osteoartrósicos submetidos à artroplastia total do quadril. Revista Brasileira de Ortopedia, 47, 498-504. https://doi.org/10.1590/S0102-36162012000400017

[4] Paiement, G.D. and Mendelsohn, C. (1997) The Risk of Venous Thromboembolism in the Orthopedic Patient: Epidemiological and Physiological Data. Orthopedics, 20, 7-9.

[5] Scolaro, J.A., Taylor, R.M. and Wigner, N.A. (2015) Venous Thromboembolism in Orthopaedic Trauma. Journal of the American Academy of Orthopaedic Surgeons, 23, 1-6. https://doi.org/10.5435/JAAOS-23-01-1 
[6] Kearon, C., Kahn, S.R., Agnelli, G., Goldhaber, S., Raskob, G.E. and Comerota, A.J. (2008) Antithrombotic Therapy for Venous Thromboembolic Disease: American College of Chest Physicians Evidence-Based Clinical Practice Guidelines (8th Edition). Chest, 133, 454S-545S. https://doi.org/10.1378/chest.08-0658

[7] Shaieb, M.D., Watson, B.N. and Atkinson, R.E. (1999) Bleeding Complications with Enoxaparin for Deep Venous Thrombosis Prophylaxis. The Journal of Arthroplasty, 14, 432-428. https://doi.org/10.1016/S0883-5403(99)90098-0

[8] Patterson, B.M., Marchand, R. and Ranawat, C. (1989) Complications of Heparin Therapy after Total Joint Arthroplasty. The Journal of Bone and Joint Surgery. American Volume, 71, 1130-1134. https://doi.org/10.2106/00004623-198971080-00002

[9] Parvizi, J., Azzam, K. and Rothman, R.H. (2008) Deep Venous Thrombosis Prophylaxis for Total Joint Arthroplasty: American Academy of Orthopaedic Surgeons Guidelines. The Journal of Arthroplasty, 23, 2-5.e1. https://doi.org/10.1016/j.arth.2008.06.028

[10] Dorr, L.D., Gendelman, V., Maheshwari, A.V., Boutary, M., Wan, Z. and Long, W.T. (2007) Multimodal Thromboprophylaxis for Total Hip and Knee Arthroplasty Based on Risk Assessment. The Journal of Bone and Joint Surgery. American Volume, 89, 2648-2657. https://doi.org/10.2106/JBJS.F.00235

[11] American Academy of Orthopaedic Surgeons (2007) Clinical Guideline on Prevention of Pulmonary Embolism in Patients Undergoing Total Hip or Knee Arthroplasty [Internet]. Rosemont: AAOS.

http://almacen-gpc.dynalias.org/publico/Prevencion\%20Embolismo\%20tras\%20artr oplastia\%20AAOOS.pdf

[12] Salvati, E.A., Sharrock, N.E., Westrich, G., Potter, H.G., Valle, A.G.D. and Sculco, T.P. (2007) The 2007 ABJS Nicolas Andry Award: Three Decades of Clinical, Basic, and Applied Research on Thromboembolic Disease after THA: Rationale and Clinical Results of a Multimodal Prophylaxis Protocol. Clinical Orthopaedics, 459, 246-254. https://doi.org/10.1097/BLO.0b013e31805b7681

[13] Salvati, E.A., Sharrock, N.E. and González Della Valle, A. (2003) Current Concepts on the Pathogenesis, Diagnosis and Multimodal Prophylaxis of Thromboembolic Disease after Total Hip Arthroplasty. Minerva Ortopedica e Traumatologica, 54, 381-401.

[14] Johanson, N.A., Lachiewicz, P.F., Lieberman, J.R., Lotke, P.A., Parvizi, J., Pellegrini, V., et al. (2009) Prevention of Symptomatic Pulmonary Embolism in Patients Undergoing Total Hip or Knee Arthroplasty. The Journal of Bone and Joint Surgery. American Volume, 17, 183-196. https://doi.org/10.5435/00124635-200903000-00007

[15] Hardinge, K. (1982) The Direct Lateral Approach to the Hip. The Bone \& Joint Journal, 64, 17-19. https://doi.org/10.1302/0301-620X.64B1.7068713

[16] Dripps, R.D. (1963) New Classification of Physical Status. Anesthesiology, 24, 111.

[17] Guia Farmacêutico Brasíndice. Andrei Publicações Médicas, Farmacêuticas e Técnicas Ltda, São Paulo. http://www.brasindice.com.br

[18] Sharrock, N.E., Gonzalez Della Valle, A., Go, G., Lyman, S. and Salvati, E.A. (2008) Potent Anticoagulants Are Associated with a Higher All-Cause Mortality Rate after Hip and Knee Arthroplasty. Clinical Orthopaedics and Related Research, 466, 714-721. https://doi.org/10.1007/s11999-007-0092-4

[19] Geerts, W.H., Pineo, G.F., Heit, J.A., Bergqvist, D., Lassen, M.R., Colwell, C.W., et al. (2004) Prevention of Venous Thromboembolism: The Seventh ACCP Confe- 
rence on Antithrombotic and Thrombolytic Therapy. Chest, 126, 338S-400S. https://doi.org/10.1378/chest.126.3_suppl.338S

[20] Geerts, W.H., Bergqvist, D., Pineo, G.F., Heit, J.A., Samama, C.M., Lassen, M.R., et al. (2008) Prevention of Venous Thromboembolism: American College of Chest Physicians Evidence-Based Clinical Practice Guidelines (8th Edition). Chest, 133, 381S-453S. https://doi.org/10.1378/chest.08-0656

[21] Falck-Ytter, Y., Francis, C.W., Johanson, N.A., Curley, C., Dahl, O.E., Schulman, S., et al. (2012) Prevention of VTE in Orthopedic Surgery Patients: Antithrombotic Therapy and Prevention of Thrombosis, 9th ed.: American College of Chest Physicians Evidence-Based Clinical Practice Guidelines. Chest, 141, e278S-e325S. https://doi.org/10.1378/chest.11-2404

[22] Raphael, I.J., Tischler, E.H., Huang, R., Rothman, R.H., Hozack, W.J. and Parvizi, J. (2014) Aspirin: An Alternative for Pulmonary Embolism Prophylaxis after Arthroplasty? Clinical Orthopaedics and Related Research, 472, 482-488.

https://doi.org/10.1007/s11999-013-3135-Z

[23] Burnett, R.S.J., Clohisy, J.C., Wright, R.W., McDonald, D.J., Shively, R.A., Givens, S.A., et al. (2007) Failure of the American College of Chest Physicians-1A Protocol for Lovenox in Clinical Outcomes for Thromboembolic Prophylaxis. The Journal of Arthroplasty, 22, 317-324. https://doi.org/10.1016/j.arth.2007.01.007

[24] Lachiewicz, P.F. and Soileau, E.S. (2006) Multimodal Prophylaxis for THA with Mechanical Compression. Clinical Orthopaedics and Related Research, 453, 225-230. https://doi.org/10.1097/01.blo.0000238861.84733.9d

[25] Ogonda, L., Hill, J., Doran, E., Dennison, J., Stevenson, M. and Beverland, D. (2016) Aspirin for Thromboprophylaxis after Primary Lower Limb Arthroplasty: Early Thromboembolic Events and 90 Day Mortality in 11459 Patients. The Bone \& Joint Journal, 98, 341-348. https://doi.org/10.1302/0301-620X.98B3.36511

[26] Pitto, R.P., Hamer, H., Heiss-Dunlop, W. and Kuehle, J. (2004) Mechanical Prophylaxis of Deep-Vein Thrombosis after Total Hip Replacement a Randomised Clinical Trial. The Journal of Bone and Joint Surgery, 86, 639-642. https://doi.org/10.1302/0301-620X.86B5.14763

[27] Della Valle, A.G., Serota, A., Go, G., Sorriaux, G., Sculco, T.P., Sharrock, N.E., et al. (2006) Venous Thromboembolism Is Rare with a Multimodal Prophylaxis Protocol after Total Hip Arthroplasty. Clinical Orthopaedics and Related Research, 443, 146-153.

[28] Pulmonary Embolism Prevention (PEP) Trial Collaborative Group (2000) Prevention of Pulmonary Embolism and Deep Vein Thrombosis with Low Dose Aspirin: Pulmonary Embolism Prevention (PEP) Trial. The Lancet, 355, 1295-1302. https://doi.org/10.1016/S0140-6736(00)02110-3

[29] Patel, V.P., Walsh, M., Sehgal, B., Preston, C., DeWal, H. and Di Cesare, P.E. (2007) Factors Associated with Prolonged Wound Drainage after Primary Total Hip and Knee Arthroplasty. The Journal of Bone and Joint Surgery, 89, 33-38. https://doi.org/10.2106/JBJS.F.00163

[30] Parvizi, J., Ghanem, E., Joshi, A., Sharkey, P.F., Hozack, W.J. and Rothman, R.H. (2007) Does "Excessive" Anticoagulation Predispose to Periprosthetic Infection? The Journal of Arthroplasty, 22, 24-28. https://doi.org/10.1016/j.arth.2007.03.007

[31] Callaghan, J.J., Warth, L.C., Hoballah, J.J., Liu, S.S. and Wells, C.W. (2008) Evaluation of Deep Venous Thrombosis Prophylaxis in Low-Risk Patients Undergoing Total Knee Arthroplasty. The Journal of Arthroplasty, 23, 20-24.

https://doi.org/10.1016/j.arth.2008.05.018 
[32] Leite, S.N. and Vasconcellos, M.P.C. (2003) Adesão à terapêutica medicamentosa: Elementos para a discussão de conceitos e pressupostos adotados na literatura. Ciênc Saúde Coletiva, 8, 775-782.

https://doi.org/10.1590/S1413-81232003000300011

[33] Bayley, E., Brown, S., Bhamber, N.S. and Howard, P.W. (2016) Fatal Pulmonary Embolism Following Elective Total Hip Arthroplasty: A 12-Year Study. The Bone \& Joint Journal, 98, 585-588. https://doi.org/10.1302/0301-620X.98B5.34996 